\title{
O que a cavidade oral pode mostrar em pacientes com COVID-19? Revisão
}

\section{integrativa da literatura}

\author{
What oral cavity can show in COVID-19 patients? Literature integrative review \\ ¿Qué puede mostrar la cavidad bucal en pacientes de COVID-19? Revisión integrativa de la \\ literatura
}

Recebido: 19/03/2021 | Revisado: 26/03/2021 | Aceito: 27/03/2021 | Publicado: 04/04/2021

Thiago de Amorim Carvalho

ORCID: https://orcid.org/0000-0003-1153-0931

Centro Universitário de Patos de Minas, Brasil

E-mail: thiagocarvalho@unipam.edu.br

Dayviddy Lucas Magalhães Silva

ORCID: https://orcid.org/0000-0002-6708-2659

Centro Universitário de Patos de Minas, Brasil

E-mail: dayviddylms@unipam.edu.br

Laura Cesário Oliveira

ORCID: https://orcid.org/0000-0002-7689-5061

Centro Universitário de Patos de Minas, Brasil

E-mail: lauracesario@unipam.edu.br

Fabrício Campos Machado

ORCID: https://orcid.org/0000-0003-4603-8795 Centro Universitário de Patos de Minas, Brasil

E-mail: fabriciocampos@unipam.edu.br

Rodrigo Soares de Andrade

ORCID: https://orcid.org/0000-0001-6114-0929

Centro Universitário de Patos de Minas, Brasil

E-mail: rodrigosa@unipam.edu.br

Veridiana Resende Novais

ORCID: https://orcid.org/0000-0001-9017-2946

Universidade Federal de Uberlândia, Brasil

E-mail: veridiana.novais@ufu.br

\begin{abstract}
Resumo
A COVID-19, doença pandêmica, não possui sua patogenia completamente esclarecida embora se trate de uma infecção polissistêmica que pode inclusive apresentar manifestações orais, devido à capacidade do vírus SARS-CoV-2 se ligar a enzima conversora de angiotensina 2 (ACE-2). A presente revisão integrativa da literatura tem como objetivo identificar as principais manifestações orais da infecção pelo vírus SARS- CoV2 e buscar estabelecer se há ou não relação entre o surgimento das manifestações bucais com o quadro sistêmico e prognóstico desse paciente. Para realização desse trabalho utilizou-se a base de dados Pubmed, com a seleção de 11 artigos, após aplicação de critério de inclusão e exclusão, baseados na estratégia PRISMA-ScR para análise qualitativa. Os resultados demonstram que as manifestações orais mais prevalentes associadas à COVID-19 são a candidose oral e as lesões ulceradas, mas também podem surgir lesões herpéticas, gengivite descamativa, periodontite necrosante, entre outras. Além disso, apresentam o comprometimento gustativo como o sintoma mais comum sendo a disgeusia o mais predominante. E embora alguns pesquisadores já relatem associação entre manifestações mais graves da doença e disseminação das lesões orais, mais estudos clínicos e randomizados são necessários para elucidar de maneira definitiva a relação entre as manifestações orais e o prognóstico da síndrome sistêmica da COVID-19.
\end{abstract}

Palavras-chave: Coronavírus; Odontologia; Estomatologia.

\begin{abstract}
COVID-19, a pandemic disease, does not have its pathogenesis completely clarified although it is a polysystemic infection that may even present oral manifestations, due to the ability of the SARS-CoV-2 virus to bind to the angiotensin-converting enzyme 2 (ACE-2). This integrative literature review aims to identify the main oral manifestations of SARS-CoV2 virus infection and seek to establish whether or not there is a relationship between the appearance of oral manifestations and the systemic condition and prognosis of this patient. To perform this work, the Pubmed database was used, with the selection of 11 articles, after applying the inclusion and exclusion criteria, based on the PRISMA-ScR strategy for qualitative analysis. The results show that the most prevalent oral manifestations associated with COVID-19 are oral candidiasis and ulcerated lesions, but herpetic lesions, desquamative gingivitis,
\end{abstract}


necrotizing periodontitis, among others, can also occur. In addition, they present taste impairment as the most common symptom, with dysgeusia being the most prevalent. And although some researchers already report an association between more severe manifestations of the disease and dissemination of oral lesions, more clinical and randomized studies are needed to definitively elucidate the relationship between oral manifestations and the prognosis of the systemic syndrome of COVID-19.

Keywords: Coronavirus; Dentistry; Oral medicine.

\section{Resumen}

COVID-19, una enfermedad pandémica, no tiene su patogenia completamente aclarada aunque es una infección polisistémica que incluso puede presentar manifestaciones orales, debido a la capacidad del virus SARS-CoV-2 de unirse a la enzima convertidora de angiotensina 2 (ACE-2). Esta revisión integradora de la literatura tiene como objetivo identificar las principales manifestaciones orales de la infección por virus SARS-CoV2 y buscar establecer si existe o no relación entre la aparición de manifestaciones orales y la condición sistémica y pronóstico de este paciente. Para realizar este trabajo se utilizó la base de datos Pubmed, con la selección de 11 artículos, luego de aplicar los criterios de inclusión y exclusión, basados en la estrategia PRISMA-ScR para análisis cualitativo. Los resultados muestran que las manifestaciones orales más prevalentes asociadas a COVID-19 son candidiasis oral y lesiones ulceradas, pero también pueden ocurrir lesiones herpéticas, gingivitis descamativa, periodontitis necrotizante, entre otras. Además, presentan la alteración del gusto como síntoma más común, siendo la disgeusia el más prevalente. Y aunque algunos investigadores ya reportan una asociación entre las manifestaciones más graves de la enfermedad y la diseminación de las lesiones orales, se necesitan más estudios clínicos y aleatorizados para dilucidar definitivamente la relación entre las manifestaciones orales y el pronóstico del síndrome sistémico de COVID-19.

Palabras clave: Coronavirus; Odontologia; Medicina oral.

\section{Introdução}

A pandemia do novo coronavírus (COVID-19) que trouxe um cenário global de isolamento e distanciamento social é causada pelo vírus de RNA SARS-CoV-2 que se tratado sétimo coronavírus com potencial para infectar humanos (Amorim dos Santos et al., 2021; Sinjari et.al, 2020; Oliveira et al., 2020). A síndrome desencadeada pela infecção por esse vírus é caracterizada por fadiga, febre, tosse seca, mialgias, dor de garganta, dificuldades respiratórias e sintomas respiratórios que variam de leves até uma pneumonia fulminante e dificuldade respiratória potencialmente letal (Amorim dos Santos et al.,2021; Brandão et al., 2021). Além disso, o paciente pode apresentar uma série de complicações sistêmicas (Iser et al., 2020; Brandão et al., 2021), como, dano cardíaco agudo, insuficiência renal aguda, disgeusia, anosmia entre outros (Brandão et al., 2021).

A respeito da epidemiologia da COVID-19, infere-se que a pandemia tem uma característica agressiva, haja vista os dados globais sugerem que o número de casos dobra no período de quatro dias o que torna a situação ainda mais alarmante. A gravidade da doença parece estar intimamente ligada à idade e condições sistêmicas preexistentes, sendo que indivíduos com idade superior a 55 anos apresentam sintomas mais severos da doença (Uddin et al., 2020). Em relação à vacinação da população, embora pareça que seja o meio mais eficaz de reduzir o ritmo de contágio e já haver disponibilidade de vacinas com tecnologias distintas (Brasil, 2021), no Brasil, a tentativa de evitar o colapso do Sistema Universal de Saúde (SUS) não teve o efeito esperado em estados com déficit de assistência pública (Câmara et al., 2020), o que contribui com a morosidade do processo de imunização e possibilita a perpetuação de uma morbi-mortalidade preocupante. Em países como Estados Unidos, com ações políticas baseadas na ciência percebe-se uma redução da taxa de contágio e uma velocidade de imunização alta onde cerca de 12.928.749 pessoas já receberam pelo menos 1 dose da vacina já no primeiro mês de campanha (Painter et al., 2021).

O SARS-CoV-2 possui a capacidade de se ligar ao receptor da enzima conversora de angiotensina 2 (ACE2), que é detectado na membrana celular de vários órgãos e tecidos humanos, entre eles os pulmões, trato respiratório superior, células epiteliais da língua, glândulas salivares, sistema nervoso, entre outros. Assim, as células que possuem o receptor ACE2 tornam-se hospedeiras do vírus e ainda podem causar uma reação inflamatória. Os sinais e sintomas orais relacionados à COVID-19 são as alterações no paladar, gengivite descamativa, ulcerações orais, petéquias, xerostomia e o indivíduo fica suscetível a coinfecções, como as fúngicas oportunistas, infecção por vírus herpes simples oral recorrente (HSV-1). No 
entanto, ainda é incerto se o padrão clínico de manisfetação é resultado de uma infecção direta por SARS-CoV-2 ou uma consequência sistêmica de outros agravantes relacionados à própria resposta inflamatória ocasionada pela COVID-19 ou por fatores diversos como a hospitalização ou a queda de imunidade. (Amorim dos Santos et al., 2021; Sinjari et al., 2020; Brandão et al., 2021).

O objetivo desse estudo é identificar as principais manifestações existentes na mucosa oral de pacientes com COVID19 e suas relações com o prognóstico da infecção sistêmica.

\section{Metodologia}

Trata-se de um estudo de revisão integrativa cuja pergunta do estudo foi elaborada utilizando-se a estratégia PICO (Santos et al., 2007) em que atribui-se ao P (população) pacientes com COVID-19 que apresentam manifestações orais, I (indicador) a avaliação estomatológica na busca por lesões em mucosa e gengiva, C (controle) pacientes com COVID-19 que não apresentam manifestações bucais e $\mathrm{O}$ (desfecho) relação entre as manifestações orais e prognóstico da saúde sistêmica e quais as alterações bucais mais frequentes em indivíduos com COVID-19.

Assim sendo a pergunta do estudo é: "quais as relações entre a COVID-19 e as manifestações orais no que se diz respeito às áreas acometidas na cavidade bucal e o prognóstico sistêmico dos pacientes com a infecção?"

A pesquisa foi realizada seguindo o Guia PRISMA (Principais Itens para Relatar Revisões sistemáticas e Metaanálises) (Galvão et al., 2015) na base de dados PUBMED/MEDLINE. Foram utilizadas as palavras chaves de busca "oral manifestation" e "COVID-19" com o uso do operador booleano "and". Os critérios de inclusão dos artigos na pesquisa foram os relatos de caso, revisões sistemáticas, séries de casos, carta ao editor e revisão narrativa compreendidos nos anos de $2020 \mathrm{e}$ 2021. Apenas artigos em inglês e disponíveis na íntegra foram considerados. Como critério de exclusão foram artigos com apenas resumos disponíveis, opiniões de especialistas, artigos em outros idiomas que não o inglês.

\section{Resultados}

Para que fique clara a trajetória metodológica utilizada para a revisão integrativa, os critérios de elegibilidade dos artigos, associados ao fluxo de aplicação dos critérios de inclusão e exclusão estão demonstrados na Figura 1. 
Figura 1 - Fluxograma do processo de seleção dos estudos primários adaptado do Preferred Reporting Items for MetaAnalyses (PRISMA-ScR).
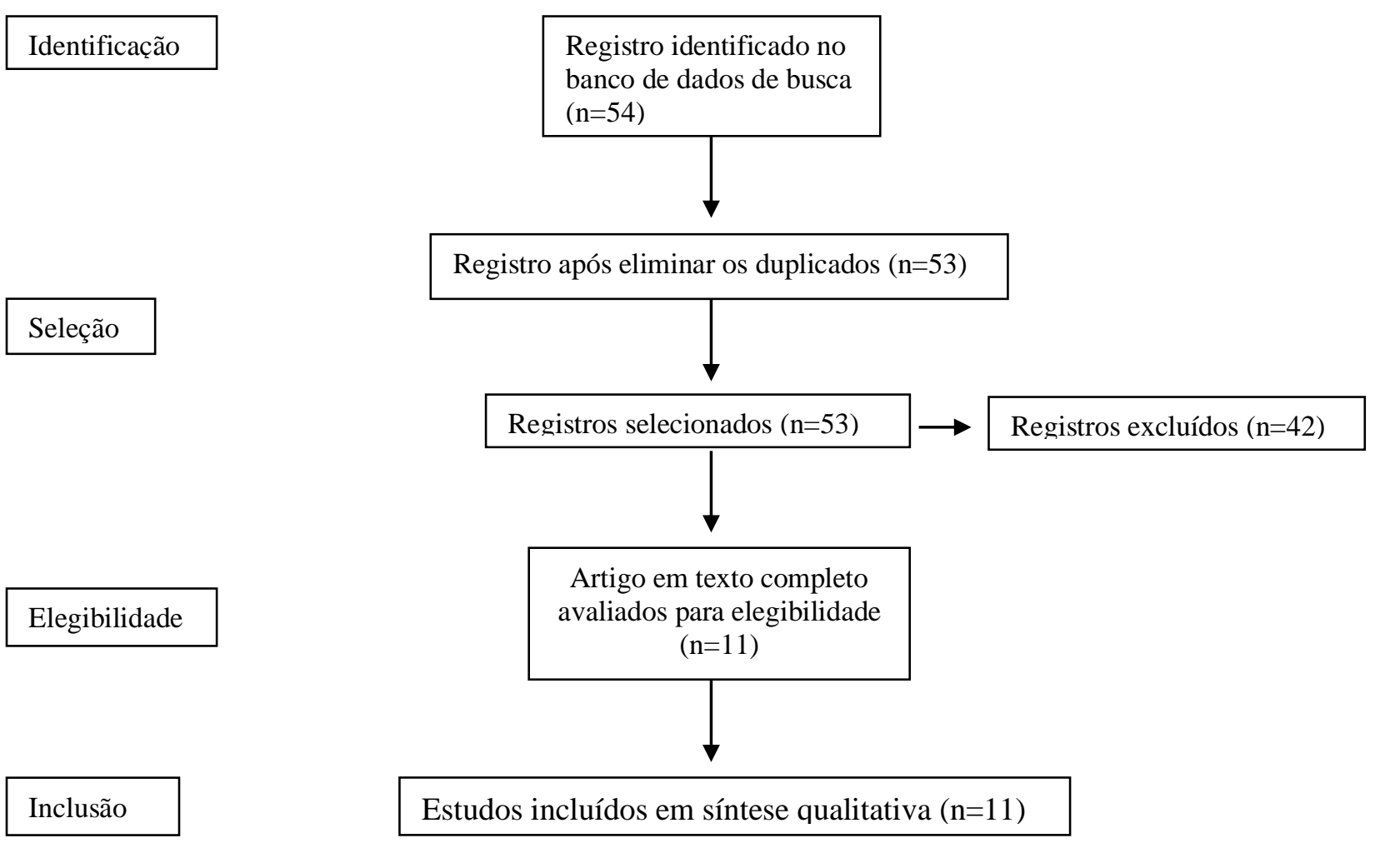

Fonte: Autores.

Conforme demonstrado na Figura1, a busca de dados resultou na identificação de 54 artigos potencialmente elegíveis todos encontrados na base de dados PubMed. Não foram utilizadas outras fontes de publicações. A leitura dos títulos resultou em 53 artigos selecionados, número que foi reduzido para 11, após a exclusão de 42 artigos baseada na leitura dos resumos. Dessa maneira 11 estudos foram selecionados para a pesquisa, nos quais constam uma revisão sistemática, uma revisão de literatura, um relato de caso, cinco estudos de série de casos e três cartas ao editor.

Após seleção, os artigos foram caracterizados de acordo com os principais resultados e as manifestações orais de COVID-19 encontradas, e a caracterização está disponível no Quadro 1. 
Quadro 1 - Caracterização do estudo, segundo autor principal, ano de publicação, periódico, objetivos, metodologia, principais resultados e manifestações orais da COVID-19 apresentados.

\begin{tabular}{|c|c|c|c|c|c|}
\hline Autor/Ano & Periódico & Objetivo & Metodologia & Principais Resultados & $\begin{array}{l}\text { Manifestações } \\
\text { (COVID-19) }\end{array}$ \\
\hline $\begin{array}{l}\text { Amorim dos } \\
\text { Santos et al., } \\
2021 .\end{array}$ & $\begin{array}{l}\text { Journal Of } \\
\text { Dental } \\
\text { Research }\end{array}$ & $\begin{array}{l}\text { Resumir as } \\
\text { evidências } \\
\text { sobre a } \\
\text { prevalência de } \\
\text { sinais r e } \\
\text { sintomas orais } \\
\text { em paciente } \\
\text { com COVID- } \\
19\end{array}$ & $\begin{array}{l}\text { Revisão } \\
\text { Sistemática, } 40 \\
\text { estudos } \\
\text { incluídos, } \quad 6 \\
\text { bancos de dados } \\
\text { consultados e } \\
\text { literatura cinza. }\end{array}$ & $\begin{array}{l}\text { Demonstra incerteza sobre } \\
\text { a verdadeira etiologia das } \\
\text { manifestações orais pela } \\
\text { COVID-19 que podem } \\
\text { surgir por coinfecções e } \\
\text { reações adversas, não sendo } \\
\text { próprias do vírus }\end{array}$ & $\begin{array}{l}\text { Estomatite aftosa, Ulcerações, } \\
\text { Gengivite descamativa, } \\
\text { Lesões herpéticas, Candidose, } \\
\text { Petéquias, Eritema } \\
\text { multiforme, lesões em língua, } \\
\text { bolhas, máculas, ageusia, } \\
\text { disgeusia e hipogeusia }\end{array}$ \\
\hline $\begin{array}{l}\text { Sinadinos \& } \\
\text { Shelswell, } \\
2020 .\end{array}$ & $\begin{array}{l}\text { Evidence- } \\
\text { Based } \\
\text { Dentistry }\end{array}$ & $\begin{array}{l}\text { Relatar } \\
\text { pacientes com } \\
\text { COVID-19 } \\
\text { que } \\
\text { apresentam } \\
\text { ulceração oral } \\
\text { ou bolhas } \\
\end{array}$ & $\begin{array}{l}\text { Série de casos, } 3 \\
\text { casos no total }\end{array}$ & 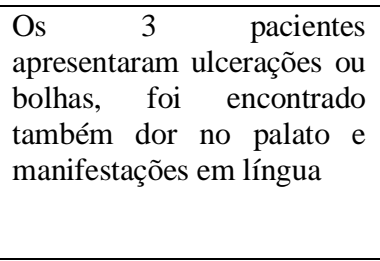 & $\begin{array}{l}\begin{array}{l}\text { Lesões de } \\
\text { eritema }\end{array} \\
\text { hipogerpes simples, } \\
\text { multiforme, }\end{array}$ \\
\hline $\begin{array}{l}\text { Sarode et al., } \\
2021 .\end{array}$ & $\begin{array}{l}\text { Medical } \\
\text { Hypotesis }\end{array}$ & $\begin{array}{l}\text { Sustentar } \\
\text { bases para a } \\
\text { comparação } \\
\text { da } \\
\text { anemia/hemól } \\
\text { ise com os } \\
\text { sintomas orais } \\
\text { da COVID-19 }\end{array}$ & \begin{tabular}{lr}
\multicolumn{2}{l}{ Carta } \\
cerca editor, \\
artigos de 11 \\
citados & base \\
referências & nas
\end{tabular} & $\begin{array}{l}\text { Acredita-se diante do } \\
\text { exposto, que, a anemia } \\
\text { através da hemólise, pode } \\
\text { causar a manifestação de } \\
\text { sintomas e manifestações } \\
\text { orais }\end{array}$ & $\begin{array}{l}\text { Ulcerações, eritema mucoso. } \\
\text { Alterações em língua } \\
\text { presentes, ageusiadisgeusia e } \\
\text { hipogeusia }\end{array}$ \\
\hline $\begin{array}{l}\text { Iranmanesh } \\
\text { "'et al., } 2021 .\end{array}$ & $\begin{array}{l}\text { Dermathologi } \\
\text { ctherapy }\end{array}$ & $\begin{array}{lr}\text { Descrever } & \text { a } \\
\text { partir } & \text { de } \\
\text { revisão } & \text { de } \\
\text { literatura, as } \\
\text { principais } \\
\text { lesões orais da } \\
\text { COVID-19 }\end{array}$ & $\begin{array}{l}\text { Revisão } \quad \text { de } \\
\text { literatura, } \\
\text { artigos } \\
\text { (Pubmed, } \\
\text { Scholar google) } \\
12 / 9 \text { até } 07 / 20 .\end{array}$ & 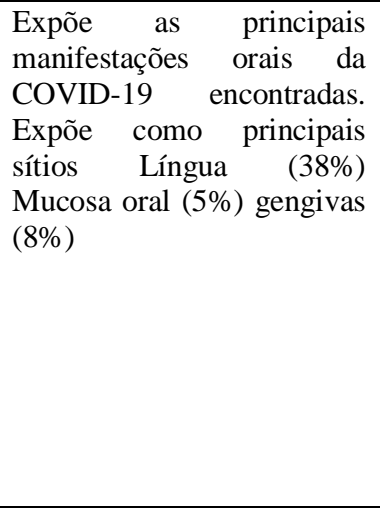 & $\begin{array}{c}\text { Estomatite aftosa, lesões } \\
\text { herpetiformes, candidose, } \\
\text { lesões orais tipo kawasaki, } \\
\text { mucosite, erupção por drogas, } \\
\text { doença periodontal } \\
\text { necrosante, angina bolhosa, } \\
\text { queilite angular, síndrome de } \\
\text { sweet atípica, síndrome de } \\
\text { Melkerson-Rosenthal, sinais e } \\
\text { sintomas e lesões } \\
\text { fundamentais oriundos dos } \\
\text { diagnósticos, língua } \\
\text { despapilada e fissurada, } \\
\text { disgeusia, halitose }\end{array}$ \\
\hline $\begin{array}{l}\text { Joseph \& } \\
\text { Prasanth, } \\
2021 .\end{array}$ & $\begin{array}{l}\text { Photodiagnosi } \\
\text { S And } \\
\text { Photodinamict } \\
\text { herapy }\end{array}$ & $\begin{array}{l}\text { Discutir um } \\
\text { novo conceito } \\
\text { de (PDT) no } \\
\text { tratamento de } \\
\text { lesões orais } \\
\text { em pacientes } \\
\text { com COVID- } \\
19\end{array}$ & $\begin{array}{lr}\text { Carta } & \text { ao editor, } \\
\text { cerca } & \text { de } 8 \\
\text { artigos } & \text { como } \\
\text { base, citados } \\
\text { nas referências }\end{array}$ & $\begin{array}{l}\text { Sugere a terapia } \\
\text { fotodinâmica como um } \\
\text { possível tratamento das } \\
\text { lesões orais pelo vírus, } \\
\text { sugerem-se mais estudo in } \\
\text { vivo para sua utilização }\end{array}$ & $\begin{array}{l}\text { Lesões vesículo bolhosas, } \\
\text { herpes simples recorrente, } \\
\text { candidose, sangramento } \\
\text { espontâneo, língua } \\
\text { despapilada/fissurada, } \\
\text { halitose e úlceras de língua }\end{array}$ \\
\hline $\begin{array}{l}\text { Riad et al., } \\
2020 .\end{array}$ & Oral Surgery & $\begin{array}{l}\text { Descrição de } \\
\text { um caso não } \\
\text { grave de } \\
\text { COVID-19 } \\
\text { Com } \\
\text { candidose oral }\end{array}$ & $\begin{array}{l}\text { Carta para o } \\
\text { editor, } 5 \text { artigos } \\
\text { referenciados e } \\
\text { exposição de } \\
\text { um caso clínico }\end{array}$ & $\begin{array}{l}\text { A principal manifestação } \\
\text { oral encontrada foi a } \\
\text { candidose } \\
\text { pseudomembranosa, que } \\
\text { em detrimento da } \\
\text { prescrição de antibióticos } \\
\text { de amplo espectro apareceu } \\
\text { como infecção oportunista }\end{array}$ & $\begin{array}{l}\text { Candidose pseudomebranosa, } \\
\text { ainda foi relatado pelo autor } \\
\text { presença de queimação e dor } \\
\text { na língua }\end{array}$ \\
\hline $\begin{array}{l}\text { Riad et al., } \\
2020 .\end{array}$ & Oral Diseases & $\begin{array}{l}\text { Expor e } \\
\text { demonstrar } \\
\text { características } \\
\text { de } 26 \\
\text { pacientes } \\
\text { confirmados } \\
\text { com a doença, } \\
\text { onde estes }\end{array}$ & $\begin{array}{l}\text { Série de casos, } \\
\text { abordando } 26 \\
\text { pacientes que } \\
\text { testaram } \\
\text { positivo para } \\
\text { COVID-19 }\end{array}$ & $\begin{array}{l}\text { Expõe a susceptibilidade da } \\
\text { mucosa oral, especialmente } \\
\text { a língua para infecção pelo } \\
\text { SARS-Cov-2, e a presença } \\
\text { de lesões ulceradas. As } \\
\text { úlceras da língua podem ser } \\
\text { manifestação direta da } \\
\text { infecção pelo vírus ou }\end{array}$ & Ulcerações, Úlceras de língua \\
\hline
\end{tabular}




\begin{tabular}{|c|c|c|c|c|c|}
\hline & & $\begin{array}{l}\text { apresentam } \\
\text { ulcerações de } \\
\text { língua }\end{array}$ & & $\begin{array}{lcc}\text { gerada pelo deficit } & \text { do } \\
\text { sistema imune } & \text { ou } \\
\text { coinfecções } & & \\
\end{array}$ & \\
\hline $\begin{array}{l}\text { Brandão et } \\
\text { al., } 2021 .\end{array}$ & Oral Medicine & $\begin{array}{lr}\text { Relatar } & 8 \\
\text { casos de } \\
\text { infecção pelo } \\
\text { Sars-Cov-2, } \\
\text { que } \\
\text { apresentam } \\
\text { úlceras } \\
\text { necróticas } \\
\text { orais e úlceras } \\
\text { aftosas, e os } \\
\text { locais alvos da } \\
\text { infecção } \\
\end{array}$ & $\begin{array}{l}\text { Série de casos, } \\
\text { abordando } 8 \\
\text { pacientes que } \\
\text { testaram } \\
\text { positivo para } \\
\text { COVID-19 }\end{array}$ & 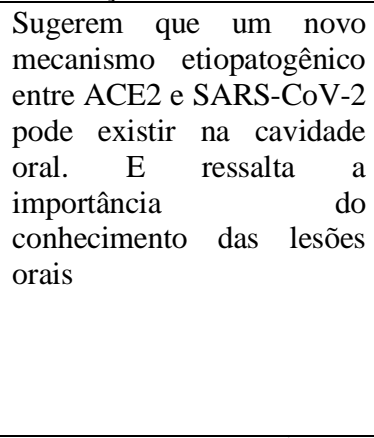 & $\begin{array}{l}\text { Ulcerações aftosas, ulcerações } \\
\text { hemorrágicas e ulcerações } \\
\text { necróticas envolvendo: } \\
\text { língua, lábios, palato e } \\
\begin{array}{l}\text { orofaringe, ageusia } \\
\text { disgeusia }\end{array}\end{array}$ \\
\hline $\begin{array}{l}\text { Hocková et } \\
\text { al., } 2021 .\end{array}$ & $\begin{array}{ll}\text { Journal } & \text { Of } \\
\text { Clinical } & \\
\text { Medicine } & \end{array}$ & $\begin{array}{lr}\text { Série } & \text { de } \\
\text { casos, } & \\
\text { associado } & \text { a } \\
\text { revisão } & \text { de } \\
\text { literatura } & \\
\text { abordando } & \\
210 \text { pacientes } \\
\text { com doença } \\
\text { grave de } \\
\text { COVID-19 }\end{array}$ & $\begin{array}{l}\text { Avaliar a } \\
\text { prevalência e as } \\
\text { características } \\
\text { dos pacientes } \\
\text { graves com } \\
\text { COVID-19 }\end{array}$ & $\begin{array}{l}\text { Demonstram que Úlceras } \\
\text { de pressão perioral, } \\
\text { candidíase oral, úlceras } \\
\text { orais herpéticas e } \\
\text { hemorrágicas } \\
\text { macroglossia aguda foram } \\
\text { as complicações } \\
\text { comumente relatadas em } \\
\text { pacientes com COVID-19 } \\
\text { em estado crítico. E ainda } \\
\text { sugerem abordagem } \\
\text { multidisciplinar no } \\
\text { monitoramento e manejo da } \\
\text { COVID-19 }\end{array}$ & $\begin{array}{l}\text { Úlceras de pressão perioral, } \\
\text { candidíase oral, úlceras orais } \\
\text { herpéticas e hemorrágicas, } \\
\text { macroglossia, disgeusia } \\
\text { também presente }\end{array}$ \\
\hline $\begin{array}{l}\text { Amorim dos } \\
\text { Santos et al., } \\
2020\end{array}$ & $\begin{array}{l}\text { International } \\
\text { Journal of } \\
\text { Infection } \\
\text { Diseases }\end{array}$ & $\begin{array}{l}\text { Relatar um } \\
\text { caso de } \\
\text { paciente com } \\
\text { diagnóstico de } \\
\text { COVID-19 } \\
\text { que apresenta } \\
\text { manifestações } \\
\text { orais } \\
\text { associadas }\end{array}$ & $\begin{array}{l}\text { Relato de caso, } \\
\text { homem branco } \\
\text { de } 67 \text { anos, } \\
\text { positivo para } \\
\text { COVID-19 }\end{array}$ & $\begin{array}{l}\text { Encontrou-se no paciente: } \\
\text { herpes simples recorrente, } \\
\text { candidose e língua } \\
\text { geográfica. O autor traz que } \\
\text { as infecções podem ocorrer } \\
\text { a partir do próprio vírus } \\
\text { através do mecanismo da } \\
\text { ACE2 ou a partir de } \\
\text { infecções oportunistas ou } \\
\text { coinfecções }\end{array}$ & $\begin{array}{l}\text { Herpes simples recorrente, } \\
\text { candidose, úlcera traumática, } \\
\text { úlcera semelhante a monilíase } \\
\text { ou candidose eritematosa, } \\
\text { petéquias, língua geográfica, } \\
\text { disgeusia, xerostomia }\end{array}$ \\
\hline $\begin{array}{l}\text { Sinjari et al., } \\
2020\end{array}$ & $\begin{array}{ll}\text { Journal } & \text { Of } \\
\text { Clinical } & \\
\text { Medicine } & \end{array}$ & $\begin{array}{l}\text { Determinar a } \\
\text { manifestação } \\
\text { oral de } \\
\text { pacientes } \\
\text { graves para } \\
\text { COVID-19 }\end{array}$ & $\begin{array}{l}\text { Estudo } \\
\text { observacional, } \\
\text { cerca de } 20 \\
\text { pacientes } \\
\text { positivos para } \\
\text { doença foram } \\
\text { submetidos a } \\
\text { questionário }\end{array}$ & $\begin{array}{l}\text { Conclui que mais estudos } \\
\text { são necessários para } \\
\text { entender melhor os } \\
\text { sintomas desse novo vírus, } \\
\text { a fim de detectar mais } \\
\text { rapidamente sua presença } \\
\text { em humanos; sugere equipe } \\
\text { multidisciplinar } \\
\text { acompanhando os pacientes } \\
\text { com COVID-19 }\end{array}$ & $\begin{array}{lr}\text { Ulcerações } & \text { orais } \\
\text { inespecíficas, } & \text { infecções } \\
\text { fúngicas } & \text { oportunistas, } \\
\text { infecções por HSV-1, erupção } \\
\text { por drogas fixas, ulcerações, } \\
\text { gengivites, hipogeusia, } \\
\text { xerostomia e disgeusia }\end{array}$ \\
\hline
\end{tabular}

Fonte: Autores.

No Quadro 1 pode-se perceber a diversidade de sinais e sintomas encontrados na cavidade oral de pessoas com COVID-19, e as metodologias dos estudos incluídos para análise qualitativa ainda se concentram em séries de casos e cartas ao editor, dada a necessidade constante de informações sobre a doença. Além disso percebe-se a necessidade de novos estudos com nível de evidência mais consistente, para que relações de inferência possam ser realizadas.

\section{Discussão}

Após análise da literatura foram encontrados diversos sinais e sintomas em cavidade oral dentre eles: ulcerações inespecíficas (Amorim dos Santos et al., 2021; Riad et al., 2020; Sarode et al., 2021; Sinjari et al., 2020), úlceras hemorrágicas (Hocková et al., 2021; Brandão et al., 2021), úlceras necróticas (Brandão et al., 2020), úlceras traumáticas, úlceras semelhante 
a monilíase ou candidose eritematosa (Amorim dos Santos et al., 2020), úlceras em língua (Riad et al., 2020; Joseph \& Prasanth., 2021), estomatite aftosa (Amorim dos Santos et al., 2021; Iranmanesh et al., 2021) gengivite descamativa (Amorim dos Santos et al., 2021), gengivite (Sinjari et al., 2020), lesões de herpes simples/lesões herpéticas (Sinjari et al., 2020; Amorim dos Santos et al., 2021; Joseph \& Prasanth., 2021; Amorim dos Santos et al., 2020; Hocková et al., 2021; Iranmanesh et al., 2021; Sinadinos \& Shelswell., 2020; lesões tipo Kawasaki (Iranmanesh et al., 2021), candidose (Sinjari et al., 2020; Amorim dos Santos et al., 2021; Amorim dos Santos et al., 2020; Joseph \& Prasanth., 2021; Iranmanesh et al., 2021; Hocková et al., 2021), candidose pseudomembranosa (Riad et al., 2020); petéquias (Amorim dos Santos et al., 2020; Amorim dos Santos et al., 2021), eritema mucoso/multiforme (Amorim dos Santos et al., 2021; Sinadinos \& Shelswell., 2020; Sarode et al., 2021), lesões em língua (Brandão et al., 2021; Riad et al., 2020; Joseph \& Prasanth., 2021; Iranmanesh et al., 2021; Sarode et al., 2021; Amorim dos Santos et al., 2021), bolhas (Joseph \& Prasanth., 2021; Amorim dos Santos et al., 2021), máculas (Amorim dos Santos et al., 2021), língua despapilada (Joseph \& Prasanth., 2021; Iranmanesh et al., 2021), língua fissurada (Joseph \& Prasanth., 2021; Iranmanesh et al., 2021), língua geográfica (Amorim dos Santos et al., 2020), mucosite (Iranmanesh et al., 2021), erupção por drogas (Sinjari et al., 2020), doença periodontal necrosante, angina bolhosa , queilite angular, síndrome de Sweet atípica, síndrome de Melkerson Rosenthal (Iranmanesh et al., 2021.), macroglossia (Hocková et al., 2021; Iranmanesh et al., 2021), ageusia (Amorim dos Santos et al., 2021; Sarode et al., 2021; Brandão et al., 2021), disgeusia (Amorim dos Santos et al., 2021; Sarode et al., 2021; Brandão et al., 2021; Hocková et al., 2021; Amorim dos Santos et al., 2020; Sinjari et al., 2020), hipogeusia (Amorim dos Santos et al., 2021; Sinadinos \& Shelswell, 2020; Sarode et al., 2021; Sinjari et al., 2020 ), xerostomia (Sinjari et al., 2020; Amorim dos Santos et al., 2020), sangramento espontâneo (Joseph \& Prasanth., 2021), halitose (Iranmanesh et al., 2021, Joseph \& Prasanth., 2021), queimação e dor na língua (Riad et al., 2020). Percebe-se que as manifestações orais mais comumente associadas à COVID-19 são lesões ulceradas e candidose oral embora não seja claro o nexo causal entre essas alterações e a COVID-19, além disso foi encontrado como sintoma mais aparente a disgeusia .

As lesões erosivas ou ulcerativas se apresentam clinicamente como lesões dolorosas com bordas irregulares sendo geralmente encontradas ou observadas na língua, mucosa labial e palato (Amorim dos Santos et al., 2021; Iranmanesh et al., 2021; Sinadinos \& Shelswell, 2020; Brandão et al., 2021; Riad et al., 2020; Hocková et al., 2021 ; Amorim dos Santos et al. (2020) ; Hocková et al., 2021) relatam que o surgimento das lesões ulcerativas ocorrem devido a presença de patógenos oportunistas ou por meio de coinfecções; podendo ser as úlceras herpéticas, as úlceras necróticas hemorrágicas e úlceras de pressão perioral. As lesões ulceradas surgem cinco dias após o agravamenntodos sintomas respiratórios e foram tratadas com antivirais e o uso de laser para terapia de potência e bioestimulação tecidual. (Brandão et al., 2021; Hocková et al., 2021). Estudos adicionais precisam ser feitos para investigar se a infecção pelo coronavírus é o fator causal para o surgimento das lesões ulceradas ou se aparecem devido a deficiência do sistema imune e as condições de higiene bucal do paciente (Brandão et al., 2021; Hocková et al., 2021).

A candidose é uma das principais manifestações orais relacionadas à COVID-19 encontrada no estudo, podendo ser na forma de placas brancas ou vermelhas. Os principais sítios de acometimento foram o palato e a língua (Riad et al., 2020). A associação da infecção aguda por COVID-19 adjunto as suas medidas terapêuticas, prescrição de antibióticos de amplo espectro e seu uso prolongado, deficiência do sistema imune, e declínio da higiene oral seriam os principais fatores que levariam ao surgimento da candidose, (Hocková et al., 2021; Amorim dos Santos et al., 2021; Amorim dos Santos et al., 2020; Riad et al., 2020). Os artigos estudados mostraram que para o tratamento dessa infecção os pacientes utilizaram medicamentos como fluconazol sistêmico ou nistatina tópica de acordo com a gravidade e a superfície da lesão (Hocková et al., 2021). Vale enfatizar que infecções como a candidose podem se tornar um risco de vida se agravadas (Hocková et al., 2021; Riad et al., 2020).

Os estudos demonstram que o comprometimento gustativo foi à manifestação oral mais comum em pacientes testados 
positivos para o vírus SARS-CoV-2 sendo que cerca de 38\% desses pacientes apresentam disgeusia que é uma distorção qualitativa da percepção do paladar (Amorim dos Santos et al., 2021; Sarode et al., 2021). As pesquisas mostram que isso ocorre devido a ACE2 ser o principal receptor do vírus SARS-CoV2 e essa enzima possuir alta expressão nas glândulas salivares e nas células epiteliais da língua, favorecendo para o surgimento da disgeusia, mas também pode causar ulcerações orais e necrose superficial da mucosa oral. (Brandão et al., 2021; Hocková et al., 2021). Assim, a disgeusia pode ser um marcador clínico da doença para os cirurgiões dentistas que trabalham na linha de frente (Brandão et al., 2021).

$\mathrm{Na}$ literatura analisada há poucos estudos que comprovem que a presença de lesões orais agrava o quadro sistêmico do paciente. No entanto, é importante conhecer as lesões, pois muitas delas precedem sintomas respiratórios típicos da COVID-19 além de que, o agravamento das lesões orais antecede um quadro clínico sistêmico mais sério (Brandão et al., 2021). Contudo, Iranmanesh et al. (2021) mostra que as manifestações clínicas orais e os sintomas sistêmicos da COVID-19 apareceram de forma simultânea e que existe uma relação da gravidade da doença com a presença de lesões orais mais severas e disseminadas.

A imunossupressão secundária a infecção por COVID-19 também pode ser um fator contribuinte para o surgimento de infecções orais oportunistas que dependendo da condição sistêmica do paciente tornam-se sinérgicas com quadros patológicos potencialmente fatais (Riad et al., 2020; Iranmanesh et al., 2021; Brandão et al., 2021)

Mais estudos são necessários para entender a patogenia do vírus e as suas implicações sistêmicas, embora note-se que a maioria das pesquisas apresenta conclusões controversas sobre o surgimento das manifestações orais. No entanto, é necessário que o exame bucal dos pacientes com COVID-19 não seja negligenciado então é fundamental que haja um atendimento multidisciplinar para garantir uma melhora da qualidade de vida desses pacientes. (Amorim dos Santos et al., 2021; Sinjari et al., 2020; Brandão et al., 2021).

\section{Conclusão}

Baseado no estudo dos 11 artigos utilizados para produzir a revisão integrativa nota-se que úlceras orais e suas variações, a candidose e a disgeusia são as manifestações orais mais presentes em pacientes com testes positivos para a COVID-19, acometendo principalmente os sítios da língua, mucosa labial e palato. Acredita-se que o surgimento das úlceras e da disgeusia seja devido à capacidade do vírus SARS-CoV-2 se ligar a enzima ACE2 e já o aparecimento da candidose devido ao comprometimento do sistema imune.

Mais estudos clínicos e randomizados precisam ser feitos para ser possível estabelecer uma real relação de causa e efeito e associar o prognóstico do paciente a presença de manifestações orais, já que estas parecem indicadores importantes para fins diagnósticos e de acompanhamento clínico. Estudos em larga escala, realizados com pacientes diagnosticados com COVID-19, com comprometimento clínico diverso, e com intervenções odontológicas, comparados com resultados de pacientes que não tenham acesso à essa intervenção, podem contribuir com a obtenção de resultados baseados em evidências científicas robustas.

\section{Referências}

Amorim Dos Santos, J., Normando, A., Carvalho da Silva, R. L., De Paula, R. M., Cembranel, A. C., Santos-Silva, A. R., \& Guerra, E. (2020). Oral mucosal lesions in a COVID-19 patient: New signs or secondary manifestations?. International journal of infectious diseases: IJID: official publication of the International Society for Infectious Diseases, 97, 326-328. https://doi.org/10.1016/j.ijid.2020.06.012

Amorim Dos Santos, J., Normando, A., Carvalho da Silva, R. L., Acevedo, A. C., De Luca Canto, G., Sugaya, N., Santos-Silva, A. R., \& Guerra, E. (2021). Oral Manifestations in Patients with COVID-19: A Living Systematic Review. Journal of Dental Research, 100(2), 141-154. https://doi.org/10.1177/0022034520957289

Brandão, T. B., Gueiros, L. A., Melo, T. S., Prado-Ribeiro, A. C., Nesrallah, A., Prado, G., Santos-Silva, A. R., \& Migliorati, C. A. (2021). Oral lesions in patients with SARS-CoV-2 infection: could the oral cavity be a target organ? Oral Surgery, Oral Medicine, Oral Pathology and Oral Radiology, 131(2), e45e51. https://doi.org/10.1016/j.oooo.2020.07.014 
Brasil., (2021). Plano Nacional de operacionalização da vacinação contra a COVID- 19. Ministério da Saúde. Brasília- DF, (2), 1-104. https://www.gov.br/saude/pt-br/media/pdf/2020/dezembro/16/plano_vacinacao_versao_eletronica.pdf

Câmara, F. P; Câmara, D. C. P.; Moreno, M.(2020). Contágio e virulência: covid-19 e a próxima pandemia / contágio e virulência. Brazilian Journal of Health Review, [S.L.], v. 3, n. 5, p. 13353-13357, 2020. http://dx.doi.org/10.34119/bjhrv3n5-160.

Galvão, T. F., Pansani, T. S. D. A., \& Harrad, D. (2015). Principais itens para relatar Revisões sistemáticas e Meta-análises: A recomendação PRISMA. Epidemiologia e Serviços de Saúde, 24(2), 335-342. https://doi.org/10.5123/S1679-49742015000200017

Gurgel, B. C. V., Borges, S. B., Borges, R. E. A., \& Calderon, P. S. (2020). COVID-19: Perspectives for the management of dental care and education. Journal of Applied Oral Science, 28, e20200358. Epub September 28, 2020.https://dx.doi.org/10.1590/1678-7757-2020-0358

Hocková, B., Riad, A., Valky, J., Šulajová, Z., Stebel, A., Slávik, R., Bečková, Z., Pokorná, A., Klugarová, J., \& Klugar, M. (2021). Oral Complications of ICU Patients with COVID-19: Case-Series and Review of Two Hundred Ten Cases. Journal of Clinical Medicine, 10(4), 581. https://doi.org/10.3390/jcm10040581

Iranmanesh, B., Khalili, M., Amiri, R., Zartab, H., \& Aflatoonian, M. (2021). Oral manifestations of COVID-19 disease: A review article. Dermatologic Therapy, 34(1), e14578. https://doi.org/10.1111/dth.14578

Iser, B. P. M., Sliva, I., Raymundo, V. T., Poleto, M. B., Schuelter-Trevisol, F., \& Bobinski, F. (2020). Definição de caso suspeito da COVID-19: uma revisão narrativa dos sinais e sintomas mais frequentes entre os casos confirmados. Epidemiologia e Serviços de Saúde, 29(3), e2020233. Epub 18 de junho de 2020.https://dx.doi.org/10.5123/s1679-49742020000300018

Joseph, B., \& Prasanth, C. S. (2021). Can Photodynamic therapy be repurposed to treat oral lesions of COVID-19? Photodiagnosis and Photodynamic Therapy, 33, 102175. Advance online publication. https://doi.org/10.1016/j.pdpdt.2021.102175

Oliveira, A. C. D., Lucas, T. C., \& Iquiapaza, R. A. (2020). O que a pandemia da covid-19 tem nos ensinado sobre adoção de medidas de precaução? Texto \& Contexto - Enfermagem, 29, e20200106. Epub 08 de maio de 2020.https://dx.doi.org/10.1590/1980-265x-tce-2020-0106

Painter, E. M.; Ussery, E. N; Patel, A.; Zell, E. R.; Moulia, D. L.; Scharf, L. G.; Lynch, M.; Ritchey, M. D.; Toblin, R. L.; Murthy, B. P.; Harris, la T. Q.; Wasley, A.; Rose, D.A.; Cohn,A.; Messonnier, N.E.. (2021). Demographic Characteristics of Persons Vaccinated During the First Month of the COVID-19 Vaccination Program - United States, December 14, 2020-January 14, 2021. Morbidity And Mortality Weekly Report, 0(5), 174-177. https://www.cdc.gov/mmwr/volumes/70/wr/pdfs/mm7005e1-H.pdf .

Riad, A., Gad, A., Hockova, B., \& Klugar, M. (2020). Oral Candidiasis in Non-Severe COVID-19 Patients: Call for Antibiotic Stewardship. Oral Surgery, 10.1111/ors.12561. Advance online publication. https://doi.org/10.1111/ors.12561

Riad, A., Kassem, I., Hockova, B., Badrah, M., \& Klugar, M. (2020). Tongue ulcers associated with SARS-CoV-2 infection: A case series. Oral Diseases, 10.1111/odi.13635. Advance online publication. https://doi.org/10.1111/odi.13635

Santos, C. M. C., Pimenta, C. A. M., \& Nobre, M. R. C., (2007). The PICO strategy for the research question construction and evidence search. Revista Latino-Americana de Enfermagem, 15(3), 508-511. https://doi.org/10.1590/S0104-11692007000300023

Sarode, G. S., Sarode, S. C., Gadbail, A. R., Gondivkar, S., Sharma, N. K., \& Patil, S. (2021). Are oral manifestations related to SARS-CoV-2 mediated hemolysis and anemia?. Medical Hypotheses, 146, 110413. https://doi.org/10.1016/j.mehy.2020.110413

Sinadinos, A., \& Shelswell, J. (2020). Oral ulceration and blistering in patients with COVID-19. Evidence-Based Dentistry, 21(2), 49. https://doi.org/10.1038/s41432-020-0100-z

Sinjari, B., D'Ardes, D., Santilli, M., Rexhepi, I., D'Addazio, G., Di Carlo, P., Chiacchiaretta, P., Caputi, S., \& Cipollone, F. (2020). SARS-CoV-2 and Oral Manifestation: An Observational, Human Study. Journal of Clinical Medicine, 9(10), 3218. https://doi.org/10.3390/jcm9103218

Uddin, M., Mustafa, F., Rizvi, T. A., Loney, T., Suwaidi, H. A., Al-Marzouqi, A., Eldin, A. K., Alsabeeha, N., Adrian, T. E., Stefanini, C., Nowotny, N., Alsheikh-Ali, A., \& Senok, A. C. (2020). SARS-CoV-2/COVID-19: Viral Genomics, Epidemiology, Vaccines, and Therapeutic Interventions. Viruses, 12(5), 526. https://doi.org/10.3390/v12050526 